\title{
Cardiac Motion Extraction Using 3D Surface Matching in Multislice Computed Tomography
}

\author{
Antoine Simon ${ }^{1}$, Mireille Garreau ${ }^{1}$, Dominique Boulmier ${ }^{2}$, Jean-Louis Coatrieux ${ }^{1}$, \\ and Herve Le Breton ${ }^{1,2}$ \\ ${ }^{1}$ LTSI, INSERM U642, Université de Rennes 1, Campus de Beaulieu, 35042 Rennes, France \\ antoine.simon@univ-rennes1.fr \\ 2 Centre Cardio-Pneumologique, CHU Pontchaillou, 35033 Rennes, France
}

\begin{abstract}
A new generation of Multislice Computed Tomography (MSCT) scanners, which allows a complete heart coverage and offers new perspectives for cardiac kinetic evaluation, is becoming widely available. A new method has been developed for the left ventricle motion analysis from dynamic MSCT images. It is based on a 3D surface matching process applied to left cavity volumes. It provides $3 \mathrm{D}$ velocity fields which can express contraction or expansion movements. First results obtained on real data show that MSCT imaging could be of great clinical interest for cardiac applications.
\end{abstract}

\section{Introduction}

The recent significant advances of spiral computed tomography, with the introduction of ultra-fast rotating gantries (-0.5s/tr-) along with multi-rows detectors, allow a huge progress toward the imaging of moving organs providing higher spatial and temporal resolutions. Non-rigid motion extraction methods are classified into three types: geometric or parametric deformable models, optical flow estimation and feature matching methods. Some of them have been applied in cardiac imaging [1,2,3], but nothing has been done for MSCT.

We propose a first method to extract elastic motion from cardiac human MSCT images. This approach is based on local 3D surface feature matching, under global constraints. First results obtained on real data show the great potential of this new imaging system for cardiac motion evaluation in non invasive imaging.

\section{Method and Results}

The proposed approach is based on surface primitive matching. It estimates local motion with global constraints providing 3D displacement vectors between two surfaces (corresponding to times $t$ and $t+1$ ) previously extracted by a 3D region growing [4]. This process requires to define: (1) the entities and their descriptive parameters; (2) the energy function for local feature matching; (3) the global matching process. The entities chosen have to regularly describe the whole surface, to be easily accessible and to enable the computation of descriptive parameters. Taking this into account, mesh nodes have been considered as entities. The mean curvature computed on the local geometry of the nodes 
has highlighted a spatial and temporal coherence and so has been retained as the main descriptive parameter. The energy function defined to match two nodes is composed of two terms: a data term (based on descriptive parameters and euclidian distance), a regularization term (based on the coordinates of a node, of its neighbours, and of their respective corresponding nodes) which allows to preserve spatio-temporal coherence. A simulated annealing is used to locally minimize the weighted summation of that two energy terms resulting in a global optimization of the correspondences.

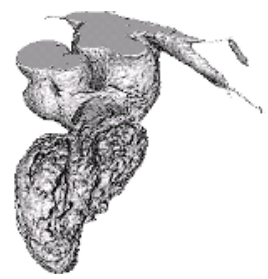

Fig. 1. Surface visualization (left anterior view)

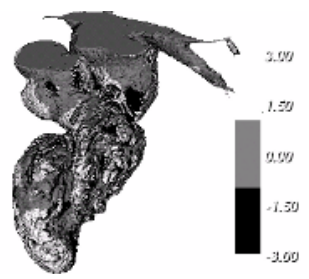

Fig. 2. Extracted motion (diastole)

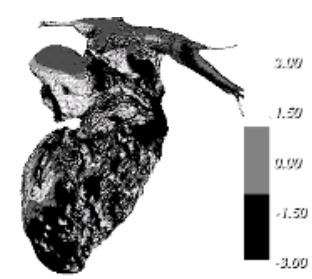

Fig. 3. Extracted motion (systole)

This method has been tested with one temporal database acquired by a Siemens SOMATOM PLUS 4 - VZ with ten volumes representing a whole cardiac cycle. The motion extraction process has been applied to two consecutive endocardial surfaces (previously smoothed (cf. Fig.1)) resulting in a set of displacement vectors defined at each surface node. From this 3D velocity field, some descriptive parameters related to cardiac kinetics can be extracted. As illustration, Fig. 2 and 3 show the motion amplitude and direction corresponding to the beginning of main cardiac phases (contraction/null or very small/expansion movements are respectively represented with white/grey/black colour). These first results show coherence between motion extracted and physiological information, and highlight the issue of contractile function evaluation.

\section{Conclusion}

A first solution has been proposed for the 3D motion extraction of the left ventricle in cardiac MSCT images. Our approach is applied on extracted volumes and provides first $3 \mathrm{D}$ velocity fields for the left cavity surface. These displacement vectors can represent accurate informations related to contraction and expansion movements. These first results confirm the great potential of MSCT imaging for cardiac applications.

Acknowledgement. This work is supported by Brittany region and the French Medical Research and Health National Institute (INSERM). The authors express their thanks to Siemens, Medical Division, France. 


\section{References}

1. Frangi et al., 2001, Three-Dimensionnal Modeling for Functionnal Analysis of Cardiac Images: A Review, IEEE Trans. on Medical Imaging, 20(1):2-25.

2. Kambhamettu C. et al., 2000, 3D non-rigid motion analysis under small deformations, Image and Vision Computing, 21(3):229-245

3. Eusemann et al., 2003, Parametric Visualization Methods for the Quantitative Assesment of Myocardial Motion, Acad. Radiol., 10:66-76

4. Guillaume et al., 2003, Segmentation de cavités cardiaques en imagerie scanner multibarettes, 12ème Forum des Jeunes Chercheurs en Génie Biologique et Médical, Nantes, France. 